This is the peer-reviewed version of the paper:

Mitic, V.V., Lazovic, G., Mirjanic, D., Fecht, H., Vlahovic, B., Arnold, W., 2020. The fractal nature as new frontier in microstructural characterization and relativization of scale sizes within space. Mod. Phys. Lett. B 34, 2050421.

https://doi.org/10.1142/S0217984920504217

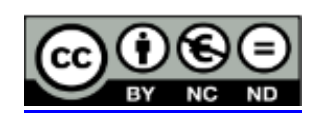

This work is licensed under the Attribution-NonCommercial-NoDerivatives 4.0 International (CC BY-NC-ND 4.0) 


\title{
The fractal nature as new frontier in microstructural characterization and relativization of scale sizes within space
}

\author{
Vojislav V. Mitic* \\ University of Nis, Faculty of Electronic Engineering, Nis, Serbia \\ Institute of Technical Sciences of SASA, Belgrade, Serbia \\ vmitic.d2480@gmail.com \\ Goran Lazovic \\ University of Belgrade, Faculty of Mechanical Engineering, Belgrade, Serbia \\ Dragoljub Mirjanic \\ ANURS, Banja Luka, Republic of Srpska, BiH \\ Hans Fecht \\ University Ulm, Germany \\ Branislav Vlahovic \\ North Carolina Central University, USA \\ Walter Arnold \\ Department of Materials Science and Engineering, \\ Saarland University, Saarbrücken, Germany
}

\begin{abstract}
Today in the age of advanced ceramic civilization, there are a variety of applications for modern ceramics materials with specific properties. Our up-to date research recognizes that ceramics have a fractal configuration nature on the basis of different phenomena. The key property of fractals is their scale-independence. The practical value is that the fractal objects' interaction and energy is possible at any reasonable scale of magnitude, including the nanoscale and may be even below. This is a consequence of fractal scale independence. This brings us to the conclusion that properties of fractals are valid on any scale (macro, micro, or nano). We also analyzed these questions with experimental results obtained from a comet, here $67 \mathrm{P}$, and also from ceramic grain and pore morphologies on the microstructure level. Fractality, as a scale-independent morphology, provides significant variety of opportunities, for example for energy storage. From the viewpoint of scaling, the relation between large and small in fractal analysis is very important. An ideal fractal can be magnified endlessly but natural morphologies cannot, what is the new light in materials sciences and space.
\end{abstract}

Keywords: Space; different scales; grains; pores; fractals.

* Corresponding author. 


\section{Introduction}

We are confident, that the exciting story about the fractals must begin from some substantial point as a part of nature and matter. Anyhow, there is the bridging correspondence between the fractal nature and the nature recognized by fractals. From these two points of view, the source and meeting point is the same, like it is the thermodynamically philosophical point of view. Sometimes we can begin by the end. The Fractals' world is everywhere around us and we are substantial part of such fractal space nature. The mathematical fractal world has been inspired by nature and Euclidean geometrical shapes. The fractal nature independently exists everywhere within structures, contact surfaces, practically, from microstructures, even on nano level and below, up to the global bulk and massive shapes.

The fractal analytic method of structural reconstruction of materials, grains and pores, in order to make possible an advanced microstructural property prognosis, is a new procedure in materials microstructural characterization. ${ }^{1}$ Electronic microscopy methods, $\frac{2}{-}$ regardless of resolution and magnification, enables one getting micrographs. This was applied on barium-titanate, $\frac{3}{-}$ silicate, refractory and other ceramics, but can be applied also to any material. Based on the grains and perimeters fractal analysis, their reconstruction is made by using the Richardson method of variable yardstick. $\stackrel{4}{-}$ It gives a more realistic picture as obtained with a Euclidean geometry frame, which replaces the role of modeling, because it gives the real micrographs shapes. The obtained micrographs, through shape reconstruction, lead to the prognosis possibility of the designed microstructural properties. $\frac{1,5}{}$

From this point of view, all modern and maximal optimized microstructure methods are faced with open questions, how to provide more flexibility in the field of the structural units (grains and pores). Their reconstruction and interrelations have the final goal to be in function of future high-level integrations and better packaging of microelectronic components, devices, and integrated circuits. This is on the way to understand that the fractal nature exists everywhere independently of distances. This opens a new view, namely that the shapes of the objects on Earth, under the telescope from space, are like the microstructures seen with the aid of a microscope.

From that aspect, the large-small relation in the light of fractal analysis is very important. An ideal fractal can be magnified endlessly while natural morphologies cannot. This is the reason why natural objects cannot be ideal fractals. The exception is maybe the Universe as whole. Our microstructures do not differ regarding fractality from macrostructures. The practical question is: What is the measurement range in which fractality can be identified? By rule, the minimum information reported by literature must be ranged in at least three orders of measured quantity magnitude.

With the development of modern physics in the early 20th century, it became clear that the Euclidean geometry, which was the successful basis of Newtonian physics is by far not sufficient to describe all visible phenomena in nature, in 
quantum physics or in the physics of deep space. ${ }^{6}$ New areas are accompanied by the development of new geometries: The Riemann and Lobachevsky geometry, and finite projective geometry. It turned out, however, that neither of the mentioned geometries are sufficient to describe some seemingly common macro-phenomena such as chaotic, turbulent and colloidal motions, crystal growth, and so on. Here and there some partial solutions have appeared in the eighties of the 20th century. These attempts were systematized by Benoit Mandelbrot in his epochal book, ${ }^{7}$ which cast a new light on the order of things in nature. It was in Ref. 7 that the term "fractal" appeared for the first time as mathematical object. The term fractal is a neologism derived from the Latin adjective fracins meaning fragmented, irregular. Due its complexity, fractal objects cannot be successfully defined without involving infinity. In Ref. 7, many examples of fractal phenomena in nature are given: they are all amorphous or hyper-complex structures such as the formation of clouds, swirling water, the movement of ocean currents, polarized light, the arrangement of stars in galaxies, vegetation, irregular forms of relief, the contours of coastlines, the alveolar configuration of lung tissues, and the like. In addition to the morphological sphere, fractals appear also in the functional sphere. For example, noise in telecommunications, free market price fluctuations, the variation in biomass of different plants and animal species or statistical performance of spoken language have fractal structure.

The complete definition of fractal cannot be given outside the framework of functional analysis and measure theory. Instead, the following weaker definition can suffice for applications in material science:

A fractal $\boldsymbol{A}$ is a subset of the complete metric space, which is invariant in relation to the union of contractive $W$ mappings, i.e. $W(\boldsymbol{A})=\boldsymbol{A}$. Thereupon, the Hausdorff dimension of this subset $D H(\boldsymbol{A})$, as a rule, is a non-integer real number.

The mapping $w$ is "contractive" if it maps the bounded original set into a set that is "smaller" in the sense of Hausdorff metric. Unlike the Euclidean metric, which determines linear distance $d(\mathbf{x}, \mathbf{y})$ between two points, the Hausdorff metric $h$ gives the distance between two non-empty sets, $A$ and $B$ as

$h(A, B)=\max \{\max \{\min (d(\mathbf{y}, \mathbf{a}), \mathbf{a} \in A), \mathbf{y} \in B\}, \max \{\min (d(\mathbf{x}, \mathbf{b}), \mathbf{b} \in B), \mathbf{x} \in A\}\}$,

If $A$ and $B$ collapses to points, the Hausdorff distance becomes Euclidean. In this sense, the Hausdorff distance $h$ is the generalization of the Euclidean distance $d$. In a similar sense, the Hausdorff or fractal dimension is the generalization of usual Euclidean dimension (also called geometric or topological dimension DT).

Figure 1 shows two sequences of geometric planar figures. The top part shows the sequences of polygons inscribed in the same circle. By doubling the number of sides, they represent increasingly in terms of the Hausdorff distance the circle (the same conclusion holds if the polygons are considered as 2D figures sequence approaching the circular disk). The bottom shows the sequence of polygons approaching a parabolic curve, which is the usual situation in approximating functions.

Another, more descriptive definition says that fractals are geometric objects having broken, fragmented, wrinkled or amorphous forms or being highly irregular 


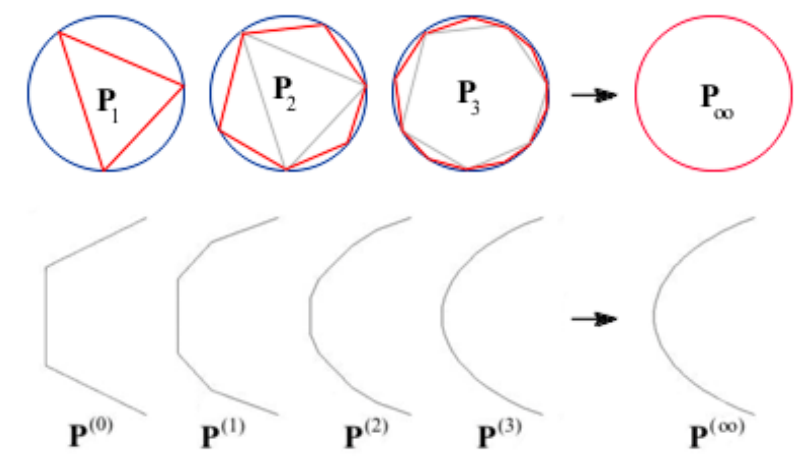

Fig. 1. Convergence in Hausdorff metric in the plane.

in some other way. In addition, some object $S$ is fractal if its fractal (Hausdorff) dimension strictly exceeds its topological dimension, i.e. if $\mathrm{DT}(S)<\mathrm{DH}(S)$.

Also, as the topological dimension DT is always a whole number ( 0 for points, 1 for curves, 2 for surfaces, 3 for solids, etc.), DH can be both integer and noninteger. For example the ideally calm pond water surface has $\mathrm{DT}=\mathrm{DH}=2$, i.e. the superficial layer of liquid molecules is (approximately) a mathematical plane. Any disturbance for example caused by a slight breeze, will make the surface geometry more complicated, still with $\mathrm{DT}=2$, but with $\mathrm{DH}>2$. As the wind grows stronger $\mathrm{DH}$ will increase until it becomes equal to 2.5. This state of water surface can be characterized as an object between a surface and a body. After a certain moment, an extremely strong wind will spread out the water droplets everywhere in the surrounding atmosphere. The form of the pond surface becomes chaotic, reaching the upper limit, $\mathrm{DH}=\mathrm{DT}=3$, transforming the planar water layer into a part of $3 \mathrm{D}$ space.

Mathematicians used to discover early fractals (without having the faintest idea what they got) as unusual particular examples. Probably the first one was published in 1883 by Georg Cantor. .8 Known as (a middle third) Cantor set or Cantor dust, it is one of the simplest, although a typical fractal set. In Fig. 2, the approximation of a Cantor set is denoted by $\boldsymbol{C}$. The fractal dimension of $\boldsymbol{C}$ can be evaluated exactly;

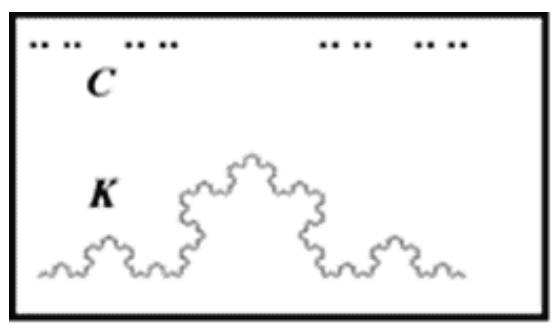

Fig. 2. Cantor dust and Koch curve. 
it is $\operatorname{DH}(\boldsymbol{C})=\log 2 / \log 3 \cong 0.63092$. So, $\boldsymbol{C}$ is an object between a point and a line. This is an explanation of the paradoxal properties of Cantor's 1883 construction: This monster has "structure" of continuum and still has zero length!

Cantor's classic set can be constructed using the so-called Iterated Function System (IFS) $)^{9}$ with two transformations $w_{1}(x)=\frac{1}{3} x, w_{2}(x)=\frac{1}{3} x+\frac{2}{3}$, joined into one single set operator $W=w_{1} \cup w_{2}$,starting by the unit interval $X=[0,1]$, so that

$$
W([0,1])=w_{1}([0,1]) \cup w_{2}([0,1])=\left[0, \frac{1}{3}\right] \cup\left[\frac{2}{3}, 1\right]
$$

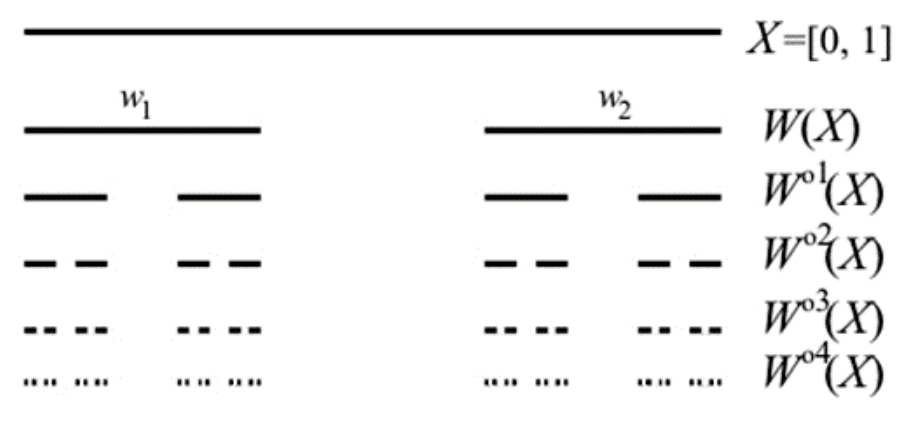

Fig. 3. Cantor set (Cantor dust) generation using IFS.

Then, by iterating,

$$
\begin{aligned}
W^{2}([0,1])= & W\left(\left[0, \frac{1}{3}\right] \cup\left[\frac{2}{3}, 1\right]\right)=w_{1}\left(\left[0, \frac{1}{3}\right] \cup\left[\frac{2}{3}, 1\right]\right) \cup w_{2}\left(\left[0, \frac{1}{3}\right] \cup\left[\frac{2}{3}, 1\right]\right) \\
= & {\left[0, \frac{1}{9}\right] \cup\left[\frac{2}{9}, \frac{1}{3}\right] \cup\left[\frac{2}{3}, \frac{7}{9}\right] \cup\left[\frac{8}{9}, 1\right] } \\
W^{3}([0,1])= & {\left[0, \frac{1}{27}\right] \cup\left[\frac{2}{27}, \frac{1}{9}\right] \cup\left[\frac{2}{9}, \frac{7}{27}\right] \cup\left[\frac{8}{27}, \frac{1}{3}\right] \cup\left[\frac{2}{3}, \frac{19}{27}\right] \cup\left[\frac{20}{97}, \frac{7}{9}\right] } \\
& \cup\left[\frac{8}{9}, \frac{25}{27}\right] \cup\left[\frac{26}{27}, 1\right]
\end{aligned}
$$

and so on (Fig. 3).

In 1875, Weierstrass came up with an example of a continuous nowhere differentiable curve. The most popular variety of such a curve is the Koch 1904 construction, the "snowflake" curve $\boldsymbol{K}$ (Fig. 2) having $\mathrm{DH}(\boldsymbol{K})=\log 4 / \log 3 \cong 1.2618$. Thus, it is not a clear curve, it is partly a surface, and despite of the fact that $\mathrm{K}$ occupies limited part of the plane, it has infinite length. It is given by the four member IFS 


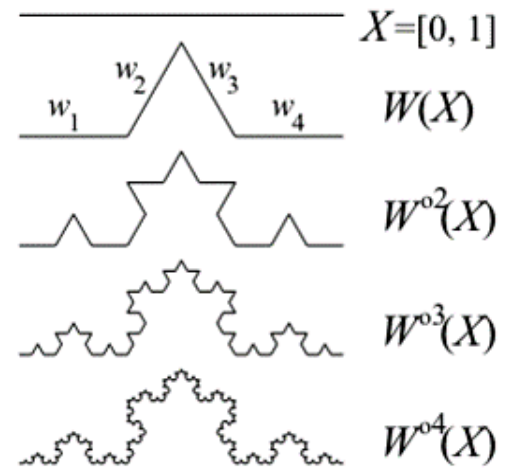

Fig. 4. Construction of Koch "snowflake" curve $K$.

acting in the $(x, y)$-plane:

$$
\begin{aligned}
w_{1}\left(\left[\begin{array}{l}
x \\
y
\end{array}\right]\right)=\left[\begin{array}{cc}
1 / 3 & 0 \\
0 & 1 / 3
\end{array}\right]\left[\begin{array}{l}
x \\
y
\end{array}\right], \quad w_{2}\left(\left[\begin{array}{l}
x \\
y
\end{array}\right]\right)=\left[\begin{array}{cc}
1 / 6 & -\frac{\sqrt{3}}{6} \\
\frac{\sqrt{3}}{6} & 1 / 6
\end{array}\right]\left[\begin{array}{l}
x \\
y
\end{array}\right]+\left[\begin{array}{c}
1 / 3 \\
0
\end{array}\right], \\
w_{3}\left(\left[\begin{array}{l}
x \\
y
\end{array}\right]\right)=\left[\begin{array}{cc}
1 / 6 & \frac{\sqrt{3}}{6} \\
-\frac{\sqrt{3}}{6} & 1 / 6
\end{array}\right]\left[\begin{array}{l}
x \\
y
\end{array}\right]+\left[\begin{array}{c}
1 / 2 \\
\frac{\sqrt{3}}{6}
\end{array}\right], \\
w_{4}\left(\left[\begin{array}{l}
x \\
y
\end{array}\right]\right)=\left[\begin{array}{cc}
1 / 3 & 0 \\
0 & 1 / 3
\end{array}\right]\left[\begin{array}{l}
x \\
y
\end{array}\right]+\left[\begin{array}{c}
2 / 3 \\
0
\end{array}\right] .
\end{aligned}
$$

The fractal dimension is now $\operatorname{DH}(\boldsymbol{K})=\ln 4 / \ln 3 \approx 1.2618595071429146159 \ldots$

Fractal models, even the simple ones, give a much more natural approximation of real physical structures than classic Euclidean geometry does. This fractal short overview inspires the ideas for fractal analysis within the whole space scale, as a joint characteristic of the total nature in all sizes reality in the matter. This characteristic is the same everywhere.

\section{Experimental}

Our experimental data are based on the results collected by some instruments of the ESA spacecraft Rosetta and Philae of the Rosetta mission ${ }^{10} \underline{-12}$ obtained on comet $67 \mathrm{P} /$ Churyumov-Gerasimenko, and on our results on the characterization of grains and pores of ceramics microstructure morphologies. In both cases we applied fractal analysis, which demonstrates common characteristics on space bodies and down to the level of nano and sub-nano structures. Figure 5 demonstrate the variety 

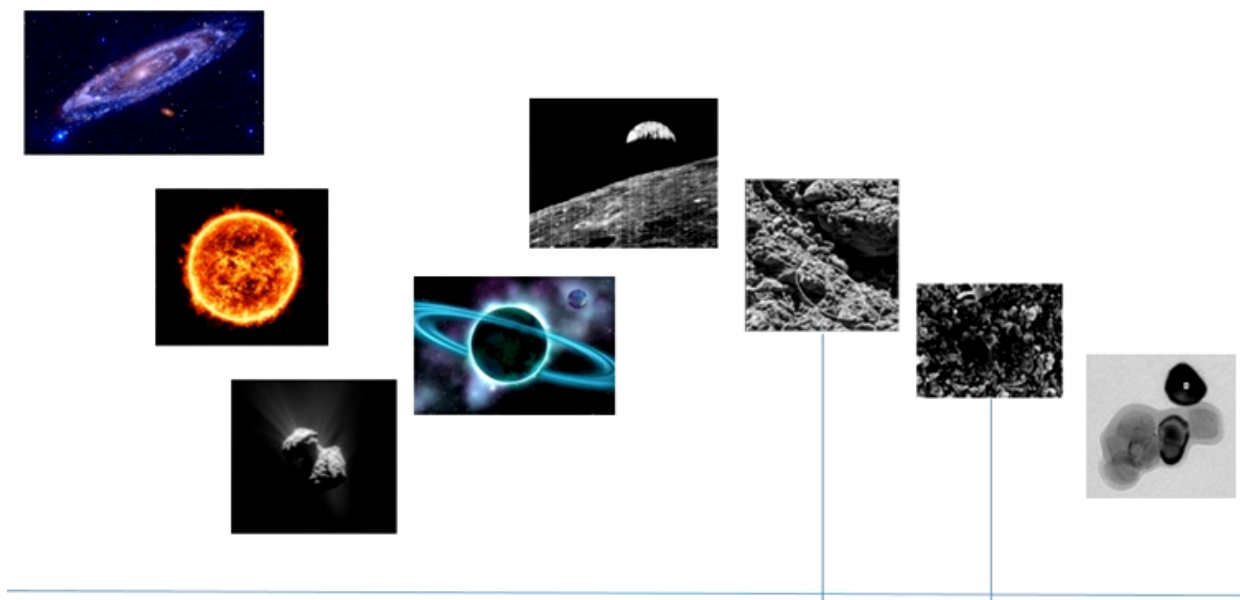

Fig. 5. Universe scales (public databases, comet, Industrial Technology Research Institute (ITRI), Taiwan).
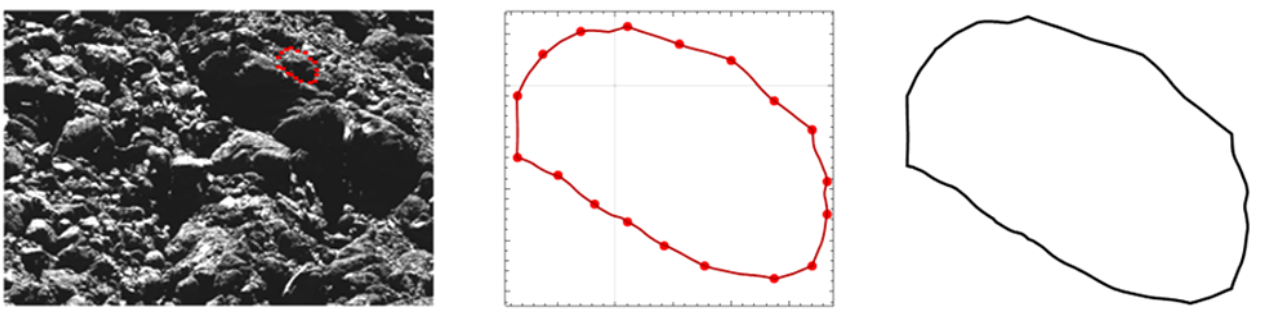

Fig. 6. Left: Comets surface microstructure at final landing place Abydos; Center and Right: Fractal analysis related parameters of some structure morphologies (Credit left-hand image: ESA/Rosetta/MPS for Ossiris Team MPS/UPD/LAM/IAA/SSO/INTA/UPM/DASP/IDA).

of different space bodies structures down to the level of sub-micro structures, which are part of the general scale of features of the universe.

A comet may represent the results of morphology structures as typical for our solar system. We analyzed the microstructural shapes at the final landing site Abydos of the Rosetta Mission lander Philae. Also we reconstructed and characterized the "grain" perimeters as shown in Fig. 6 and obtained the fractal dimension of the surface material, which is discussed in the following section.

From materials science we selected, as a base for a beginning, ceramics materials. Regarding the analysis of this extreme on the Solar system scale, grains from ceramics morphologies are studied. The ceramics samples are made of $\mathrm{BaTiO}_{3}$ with different additives like Ho, which are consolidated by standard procedure. As the starting material, commercial $\mathrm{BaTiO}_{3}$ powder (Aldrich, $99.9 \%$ purity) was used. It was treated in a planetary ball mill (Fritsch Pulverissete 5) for 10, 20, 40 and $60 \mathrm{~min}$ in an agate jar with $8 \mathrm{~mm}$ in diameter agate balls (Al2O3). The ball/sample mass 

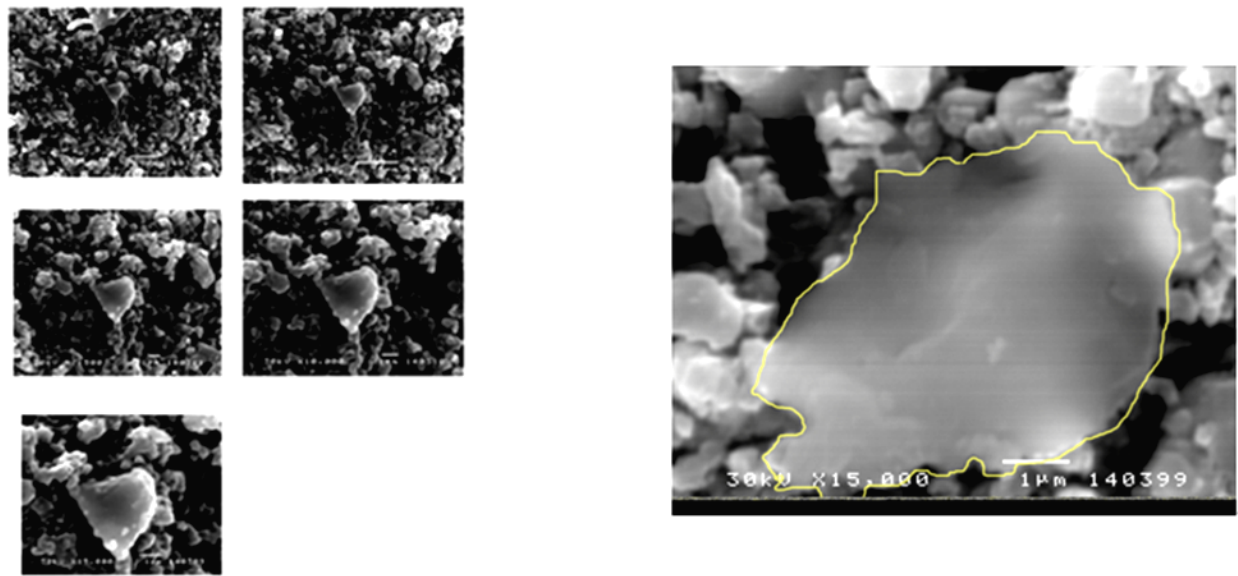

Fig. 7. Microstructures of $\mathrm{BaTiO}_{3}$-ceramics and fractals reconstructing the perimeter of the grain.

ratio was 20:1 while the tray and vial rotation speeds were 317 and $396 \mathrm{rpm}$ respectively. We carried out the microstructure analysis with five different magnifications and based on that the perimeter of the grains is shown in Fig. 7. Regarding the data collecting for grains perimeters we used small device "run-meter," which was applied for gathering the grains perimeters data. Later we used software applications for calculating the perimeters of the grains what was more effective.

On this way we have the same fractal analysis on the scale of mega bodies' sizes and micro and sub micro structures on the other extreme scale sizes.

\section{Results and Discussion}

Based on microstructure and fractal analysis from experiments, we completed the calculations of Hausdorff dimensions and disposed the related graphs. Fractal analysis of the comet's surface structure are shown in Figs. 8 and 9.

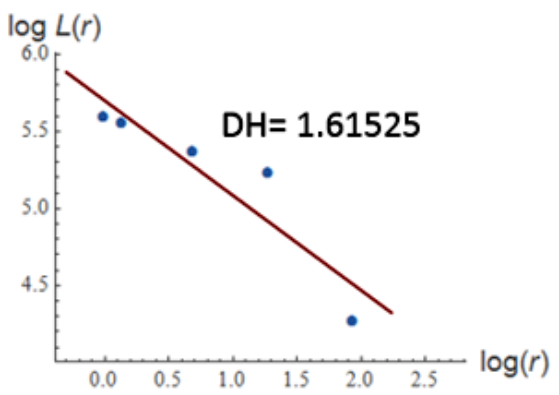

Fig. 8. The diagram of contour fractal dimension from the structures at the comet touch-down site Abydos. 

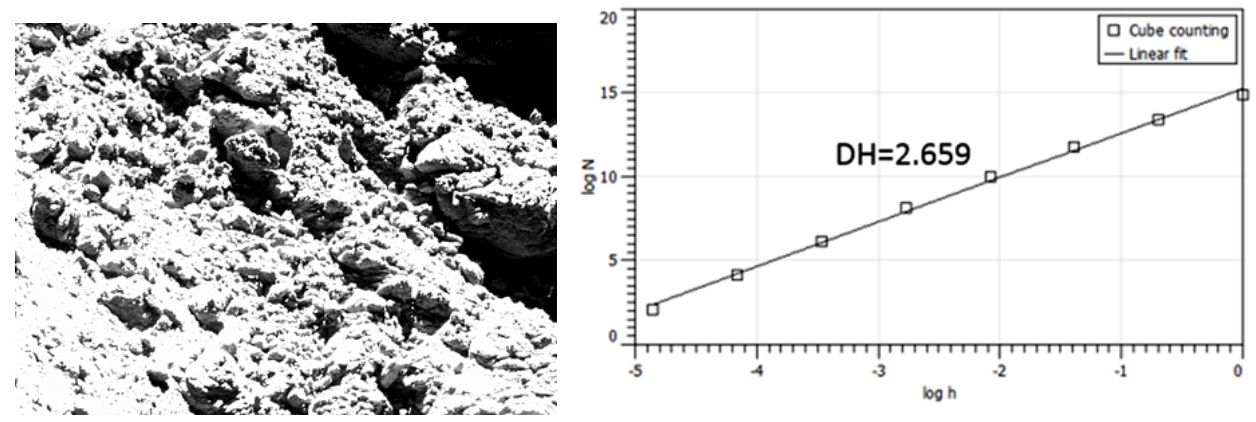

Fig. 9. Left: Comet 67P surface structure near the touch-down site Abydos (cut-out of Fig. 6 left; Right: Related surface fractal dimension.



Fig. 10. The contour fractal dimension based on $\mathrm{BaTiO}_{3}$-ceramics structures.

The results analysis from the $\mathrm{BaTiO}_{3}$-ceramics structures, taken as other extreme on the Solar system scale, is completed by contour fractal dimension and is shown in Figs. 10-12.

Regarding the data for Figs. 10-12, we used the different images of the Bariumtitanate samples consolidated with different additives quantities, sintering temperatures and pressures. So, this is principally one of all of these microstructure results, which are very similar.

Based on the sub-micro analysis of the ceramic grain surfaces, we applied Fourier analysis of the spectra roughness's, which definitely confirms the similarity of the surface nature on the micro level with surface morphology at the large space bodies like on the comet.

Based on all of these results, it is evident that fractal characteristics are a common property, both for the surface structure of small bodies of our solar system like comets, and for the microstructure of ceramics taken from SEM micrographs analysis. 


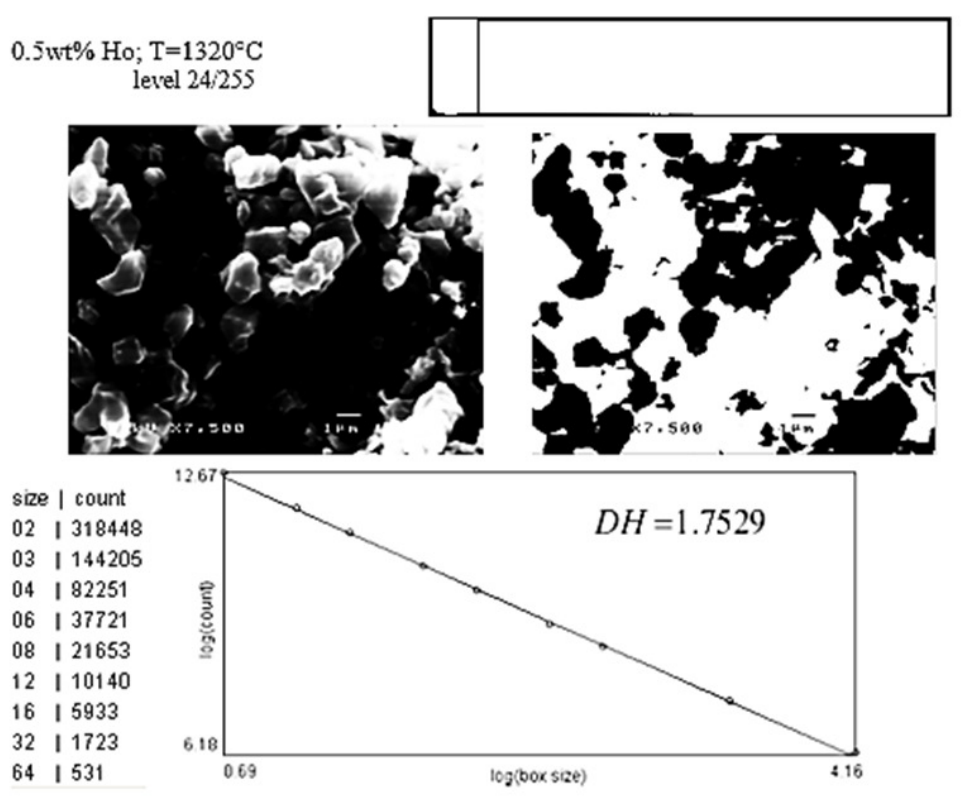

Fig. 11. $\mathrm{BaTiO}_{3}$-ceramics sample and its fractal dimension.
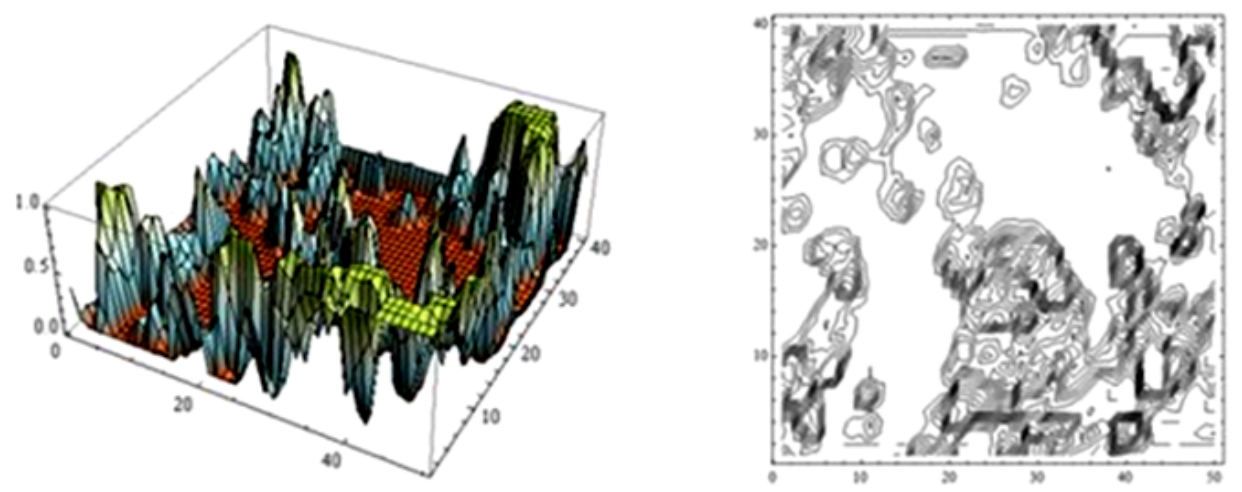

Fig. 12. Left: 3D-surface representation of the sample; Right: Level lines.

We stress that the numerical result, we may get for fractal dimension is a dimension of the picture we got and not of the sample itself. So, it may differ from the real fractal dimension for the same amount the picture differs from the original. Also, one must take into account that numerical approximations also have inherent errors. But, in spite of this, the results are quite usable in comparing two different samples and is not an obstacle in having an insight in the complicated relationship between space consolidation processes as well as on the ceramics micro level. 


\section{Conclusion and Outlook}

The relativization of different structure sizes on large scales is very important in regard to microstructural and electrophysical relations for matter properties in general. So, the fractal characterization of structures of comets is definitely the most effective bridge to the microstructure of materials on Earth, even on a nano level.

The characterization materials data, by SEM, does not have the opportunity to play the active role, without, once reconstructed microstructure shapes in the function to the microstructure properties prognosis. All available microstructure analysis tools are only passive instruments to get characterization data. The fractal structure analysis makes all of these methods lively and applicable for developing future needs. $\underline{13}$

The fractal nature offers a new approach to the ceramic structure analysis, describing prognosis and modeling the grain shapes and the relations between morphology and electrophysical properties. Also, the existence of the fractal nature of ceramic materials is completely confirmed within the electrochemical thermodynamic and fluid dynamics parameters in previous research.

This research has significance from the ceramic's microstructure consolidation prognosis fractal aspect point of view and possibility of having better insight into some internal properties. There is existing influence of ceramics grains' surface fractality plus particle dynamics in the material on the overall energy distribution, too.

Through this method and results, we are opening the fractal microstructure scale sizes new frontiers and technological processes, especially specific intergranular relations within grains surfaces in all matter.

All of these results confirm microstructure constituent's grains and pores shapes. Also, there are possibilities to analyze the Brownian motion particles phenomena. As next step, there is need for long-term scientific research on the relativization of different scale size influences within the whole nature. That is because the fractal nature is the general characteristic everywhere independently of size.

In future research, we plan to include the results, which are collected by different spacecrafts on the planets like Mars, Venus, Moon, and other solar bodies.

\section{Acknowledgment}

Gratitude to the cooperation with Industrial Technology Research Institute, Taiwan.

\section{References}

1. V. Mitic, G. Lazovic, V. Paunovic, J. R. Hwu, S.-C. Tsay, T.-P. Perng, S. Veljkovic and B. Vlahovic, J. Eur. Ceram 39(12) (2019) 3513.

2. V. V. Mitic, V. Paunovic, Lj. M. Kocic, S. Jankovic and V. Litovski, $\mathrm{BaTiO}_{3}$-ceramics microstructures new fractal frontiers, in 13th Ceramics Congress, CIMTEC 2014, CJ-1. L14, Montecatini, Italy, June 9-13, 2014. 
3. V. V. Mitic, Lj. M. Kocic, B. Jordovic and M. M. Ristic, The fractal method applied in interrelation between structure and electrical properties, in 98th Annual Meeting of the American Ceramic Society, Advanced Ceramic Materials, Electronics Division, Indianapolis, USA, 1996.

4. L. F. Richardson, General System Yearbook 6 (1961) 139.

5. V. V. Mitic, G. Lazovic, L. M. Kocic, D. Rancic, I. Antolovic and J. R. Hwu, The Voronoi Model and Fractals (National Tsing Hua University Press, 2020), in press.

6. V. V. Mitic, G. Lazovic, V. Paunovic, N. Cvetkovic, D. Jovanovic, S. Veljkovic, B. Randjelovic and B. Vlahovic, Ceramics Int. 45(7) (2019) 9679.

7. B. Mandelbrot, The Fractal Geometry of Nature (W. H. Freeman, New York, 1977).

8. G. Cantor, Math. Ann. 21(5) (1883) 545.

9. M. F. Barnsley, Constr. Approx. 2(1) (1986) 303.

10. H. Böhnhardt, J.-P. Bibring, I. Apathy, H.-U. Auster, A. E. Finzi, F. Goesmann, G. Klingelhöfer, M. Knapmeyer, W. Kofman, H. Krüger, S. Mottola, W. Schmidt, K. Seidensticker, T. Spohn and I. Wright, Phil. Trans. R. Soc. A 375 (2017) 20160248; http://dx.doi.org/10.1098/rsta.2016.0248.

11. P. Heinisch, H.-U. Auster, B. Gundlach, J. Blum, C. Güttler, C. Tubiana, H. Sierks, M. Hilchenbach, J. Biele, I. Richter and K. H. Glassmeier, A $\mathscr{E A ~ 6 3 0 ( A 2 ) ~ ( 2 0 1 9 ) ~ 8 ; ~}$ https://doi.org/10.1051/0004-6361/201833889

12. W. Arnold, H.-H. Fischer, M. Knapmeyer and H. Krüger, Jpn. J. Appl. Phys $\mathbf{5 8}$ (2019) SG0801.

13. V. V. Mitić, Lj. M. Kocić and I. Z. Mitrović, Fractals in ceramic structure, in Proc. IX World Round Table Conf. on Sintering, 1-4 September 1998, Belgrade; Advanced Science and Technology of Sintering, ed. Stojanović et al. (Kluwer Academic/Plenum Publishers, New York, 1999). 